\title{
Update on the Development of a Flutter Analysis Capability for Unconventional Aircraft Concepts using HCDstruct
}

\author{
Jesse R. Quinlan ${ }^{1}$ and Frank H. Gern ${ }^{2}$ \\ NASA Langley Research Center, Hampton, VA, 23681
}

\begin{abstract}
Following years of development, the Higher-fidelity Conceptual Design and structural optimization (HCDstruct) tool is being extended to support dynamic aeroservoelastic analysis and structural optimization for advanced aircraft concepts. These required enhancements include: the development of an aerodynamic matching routine for correcting Nastran's doublet-lattice method aerodynamics; the implementation of control surface structural models; and the implementation of support for Nastran's flutter solution sequence (SOL 145). This paper presents an update on the implementation of generalized control surface structural models and support for Nastran SOL 145.
\end{abstract}

$\begin{array}{ll}\text { CAERO1 } & =\text { Nastran DLM Panel Element } \\ \text { CAERO2 } & =\text { Nastran Slender Body Element } \\ \text { CBUSH } & =\text { Nastran Spring-Dampener System } \\ \text { CFD } & =\text { Computational Fluid Dynamics } \\ \text { CONM2 } & =\text { Nastran Concentrated Mass Element } \\ \text { CQUAD4 } & =\text { Nastran Panel Structural Element } \\ \text { DLM } & =\text { Doublet-Lattice Method } \\ \text { ECFT } & =\text { Enhanced Correction Factor Technique } \\ \text { FEA } & =\text { Finite-Element Analysis } \\ \text { FEM } & =\text { Finite-Element Model } \\ \text { FLOPS } & =\text { Flight Optimization System } \\ \text { HCDstruct } & =\text { Higher-fidelity Conceptual Design and structural optimization } \\ \text { HWB } & =\text { Hybrid Wing Body } \\ \text { LaRC } & =\text { Langley Research Center } \\ \text { OpenVSP } & =\text { Open Vehicle Sketch Pad } \\ \text { PSHELL } & =\text { Nastran Shell Properties } \\ \text { RBAR1 } & =\text { Nastran Rigid Bar Element } \\ \text { STARC-ABL } & =\text { Single-aisle Turboelectric AiRCraft with Aft Boundary Layer propulsion }\end{array}$

\section{Introduction}

\begin{abstract}
DVANCED aircraft concepts have long challenged conventional, low-order sizing methods and have in recent times Aspurred investment in physics-based methods for aircraft sizing in the conceptual design environment. Sizing analyses performed using empirical or semi-empirical methods, such as those used in the FLight OPtimization System (FLOPS)[1], are applicable only when the aircraft concept is well-represented by the training data and configuration assumptions employed by the sizing routines. Hence, sizing advanced concepts increases the demand for physics-based approaches featuring advanced computational methods, such as finite-element analysis (FEA), computational fluid dynamics (CFD), and numerical optimization. Researchers across government, academia, and industry are leveraging developments in these areas to build physics-based structural weight prediction and aeroelastic analysis tools for advanced aircraft, with several examples found in Refs. [2-11]. NASA is actively engaged in these efforts through the development of in-house software as well as through external research grants.
\end{abstract}

\footnotetext{
${ }^{1}$ Aerospace Engineer, Aeronautics Systems Analysis Branch, 1 N Dryden Street, and AIAA Member.

${ }^{2}$ Branch Head, Vehicle Analysis Branch, 1 N Dryden Street, and AIAA Member.
} 
The Higher-fidelity Conceptual Design and structural optimization (HCDstruct) toolset is a utility for advanced conceptual aircraft structural optimization and sizing. Under development for several years now, HCDstruct offers a rapid, automated means of performing static aeroelastic analysis and multidisciplinary structural design and optimization for advanced concepts using Nastran[12]. In recent times, this tool has been applied to advanced concepts such as the NASA D8 (ND8) and the Single-aisle Turboeletric AiRCraft with Aft Boundary Layer propulsion (STARC-ABL), shown in Fig. 1, which feature unique configuration elements that warrant physics-based weight estimation of the airframe structures. The current paper presents the latest enhancements to the HCDstruct toolset, which extend the core capabilities to include flutter analysis for advanced aircraft concepts. The second version of HCDstruct has been approved for public release, and this paper highlights new capabilities that will be included in the next planned sub-version release.

Following the development and demonstration of an aerodynamic matching capability for HCDstruct[2], representative control surface aerostructural models and support for Nastran's flutter solution sequence 145 (SOL $145)$ were integrated. The modeling and implementation details for these two enhancements are presented in Section II, where the control surface aerostructural models are presented in Section II.A and the flutter solution sequence support is presented in Section II.B. These developments are then exercised using a Boeing 737-200 model in Section III. Finally, the work is summarized and future efforts are described in

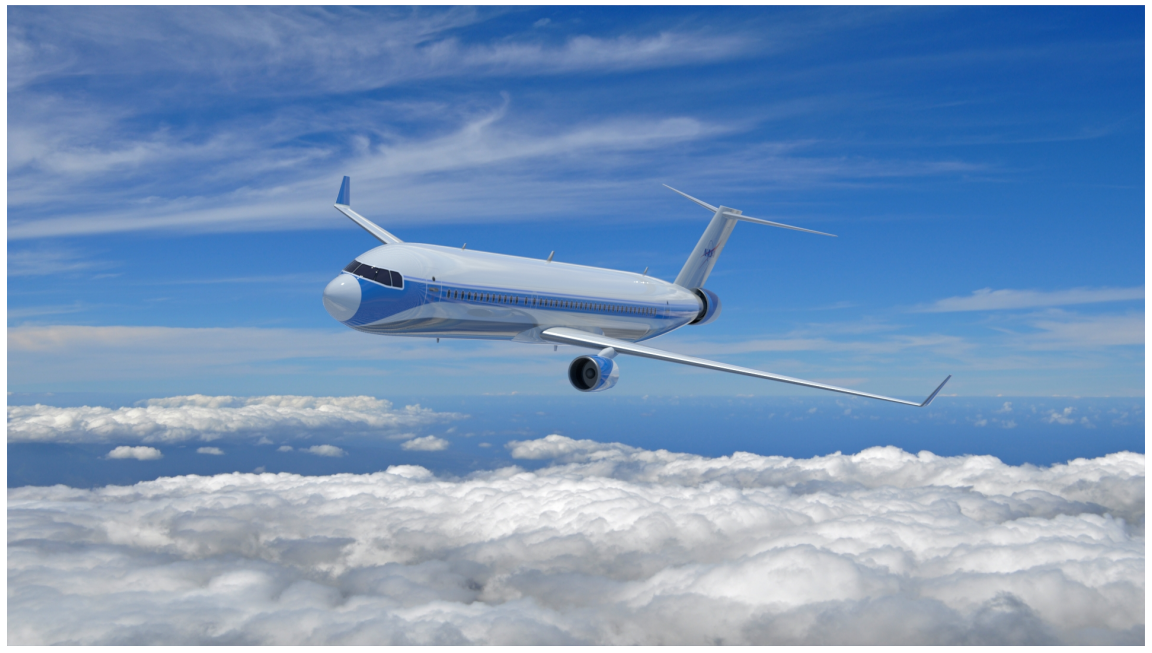

Fig. 1 NASA's STARC-ABL aircraft concept featuring an aft-mounted, boundary-layer-ingesting tailcone propulsor. (NASA Photo)

Section IV.

\section{Methodology}

HCDstruct was developed at NASA Langley Research Center (LaRC) to fill a critical analysis gap between high-level, lower-order approaches commonly used for conceptual design and the low-level, detailed, often finite-element-based optimization approaches commonly used for advanced preliminary design. Specifically, HCDstruct was developed to complement the FLOPS tool, which is a versatile, multidisciplinary suite of computer programs for conceptual and preliminary design and analysis of advanced aircraft concepts. With the regression-based sizing algorithms used by FLOPS[1, 13], model sensitivities associated with advanced configuration design elements are generally difficult to accurately predict. The detailed finite-element based sizing analyses often performed later in the design cycle, such as those for the hybrid wing body (HWB) by Boeing[14], may theoretically offer such insights, the computational resources required for these efforts often limit them to single-point design analysis. Thus, HCDstruct was developed to bridge the gap between FLOPS' regression-based sizing techniques and current state-of-the-art finite-element-based approaches. In fact, the tool has evolved to provide a means of optimizing the primary structural weight for the fuselage and wing for a given aircraft configuration using finite-element methods while only requiring FLOPS-level user data.

An overview of the static aeroelastic analysis features of HCDstruct version 2 (V2) may be found in Ref. [3] and the implementation of the Enhanced Correction Factor Technique (ECFT) $[15,16]$ for aerodynamic matching may be found in Ref. [2]; only those additional developments made in support of dynamic aeroservoelastic analysis are described in the current paper. Prior to HCDstruct V2, the control surfaces were only modeled aerodynamically. In current work, structural models for the control surfaces were implemented for use in both static and dynamic aeroservoelastic analyses. Details of this new control surface model formulation are described in Section II.A. Additionally, whereas HCDstruct 1.0 only supported static aeroelastic analysis via Nastran SOL 144, HCDstruct V2 now supports flutter analysis via Nastran SOL 145. The details of this implementation are presented in Section II.B. 


\section{A. Control Surface Modeling}

In order to properly account for aeroelastic coupling between the control surfaces and primary airframe structure, parametric structural representations of the control surfaces were developed and implemented in HCDstruct. Without these models, no control surface or combined wing-aileron flutter modes could be modeled. The objective of this development activity was to improve upon the achievable fidelity of the aeroelastic finite-element model (FEM) for flutter analyses, which prior to this work was limited since the control surfaces were only modeled aerodynamically, with corresponding forces splined directly to the primary structure.

The most direct way of providing a parametric control surface structural model amenable to flutter analysis was through the combination of rigid bars and spring-dampener systems. Specifically, Nastran RBAR1 rigid bar elements were placed near the control surface hingeline for each modeled control surface. These RBAR1 elements were then connected to the primary structure using Nastran CBUSH spring-dampener systems. With this new construct, the aerodynamic forces are now splined directly to the control surface RBAR1 elements, rather than to the primary structure. If desired, inertial effects associated with the mass of the control surfaces can be modeled using Nastran CONM2 concentrated mass elements. The CBUSH spring-dampener system can be tuned to recover the desired dynamic properties of the control surface actuators. In this way, aileron and wing-aileron flutter modes may be considered in subsequent flutter analysis using HCDstruct, and for static aeroelastic analyses, the rotational forces acting on the primary structure due to control surface deflections can also be captured.

The user has considerable flexibility in configuring these models. In the current implementation, the number of control surfaces on the main wing, the CBUSH spring stiffness and damping coefficient properties, and the RBAR1 offset distances from the primary structure connection may all be set using flags available in the standard HCDstruct input files. Two examples of such model configurations are shown in Fig. 2, where in 2(a) a more conventional aileron configuration is shown with a single outboard aileron surface modeled and in 2(b) the trailing edge of the wing is completely actuated using eight aileron surfaces, each transmitting the aerodynamic forces to the primary structure independently. Note that in these figures, only the structural model is shown; the aerodynamic panels are removed for clarity.

\section{B. Flutter Solution Sequence Integration}

In earlier versions of HCDstruct, the structural weight optimization and sizing is limited to static aeroelastic analysis via Nastran SOL 144 combined with SOL 200. However, as HCDstruct is applied to increasingly advanced concepts, especially those with higher aspect ratio wings, analyzing flutter and its effects on airframe sizing becomes ever more important. Hence, support for Nastran's flutter solution sequence, SOL 145, was recently implemented in HCDstruct. Whereas the previous version of HCDstruct, V2.1, marked the implementation of the ECFT[15, 16] for aerodynamic matching, the version of HCDstruct documented here, V2.2, represents the implementation of SOL 145 support. Details of the SOL 145 support are presented in the current section.

In order to extend HCDstruct to support Nastran SOL 145, modifications were made to several input files to provide the user complete configurability of the the flutter analysis bulk data files (BDFs). Foremost, a FLUTTER input variable was added to the HCDstruct executive input file, exec.inp, which allows the user to flag whether SOL 145 BDF input files are desired for execution by Nastran. Once the user signals in exec.inp that a flutter analysis is desired, HCDstruct then parses additional input in the aerodynamic input file, aero.inp. Within this file, the user must supply input concerning all the configuration variables shown in Table 1. With the aero.inp input data and the FLUTTER flag enabled in exec.inp, execution of HCDstruct will create all files required to perform a flutter analysis using Nastran SOL 145.

Execution of the flutter BDF files requires the patch file, hcdstruct_exec.pch, from the static aeroelastic structural optimization execution that contains the optimized shell element thicknesses. Further, the completion of the aeroelastic FEM relies on first executing the glued contact theory BDF using Nastran SOL 101, as configured in glue_exec.bdf. Hence, performing a flutter analysis using HCDstruct relies on three sequential Nastran simulations, which can be done easily by the user either manually or via a script; the representative execution flowpath is shown in Fig. 3 . 


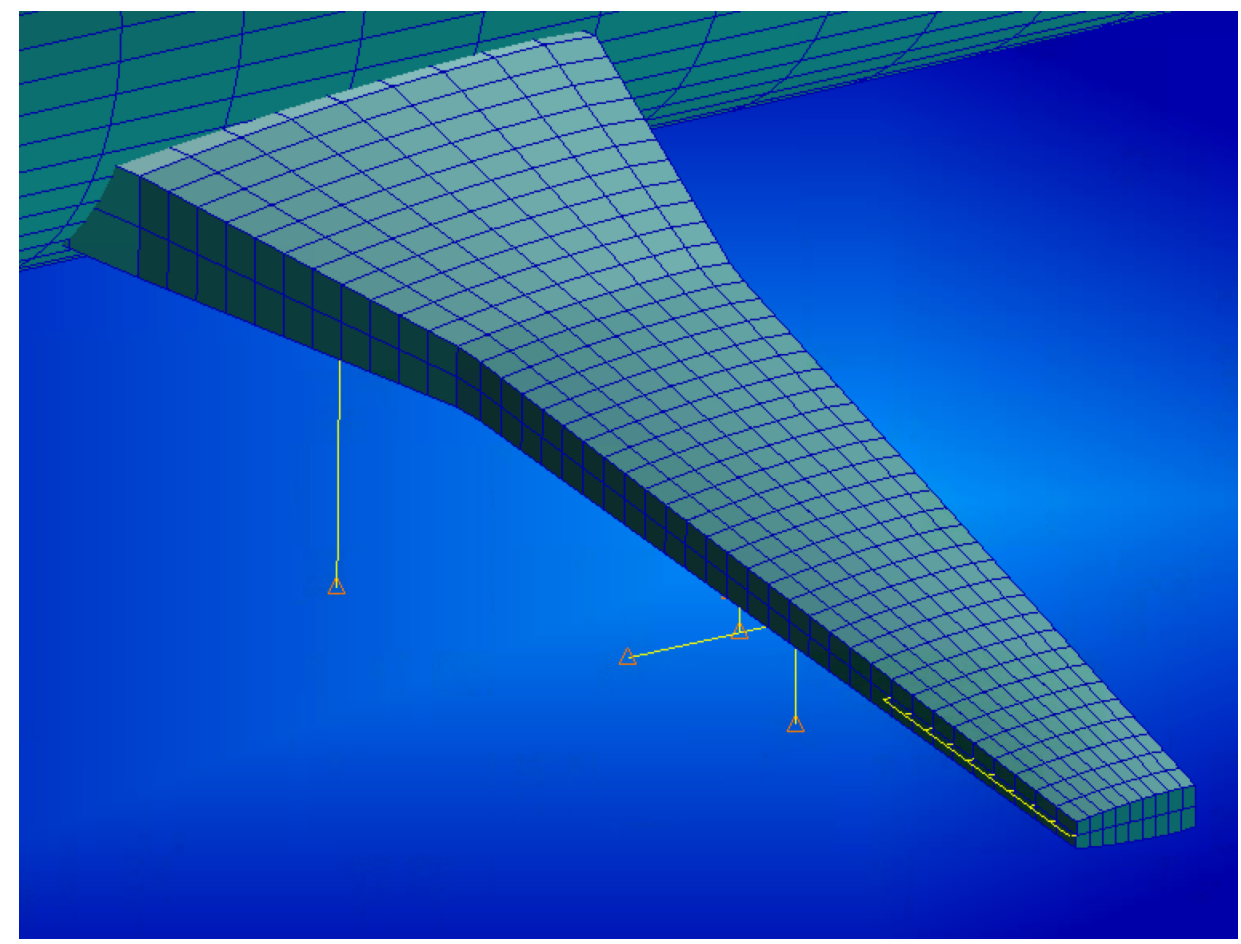

(a) Single outboard aileron example.

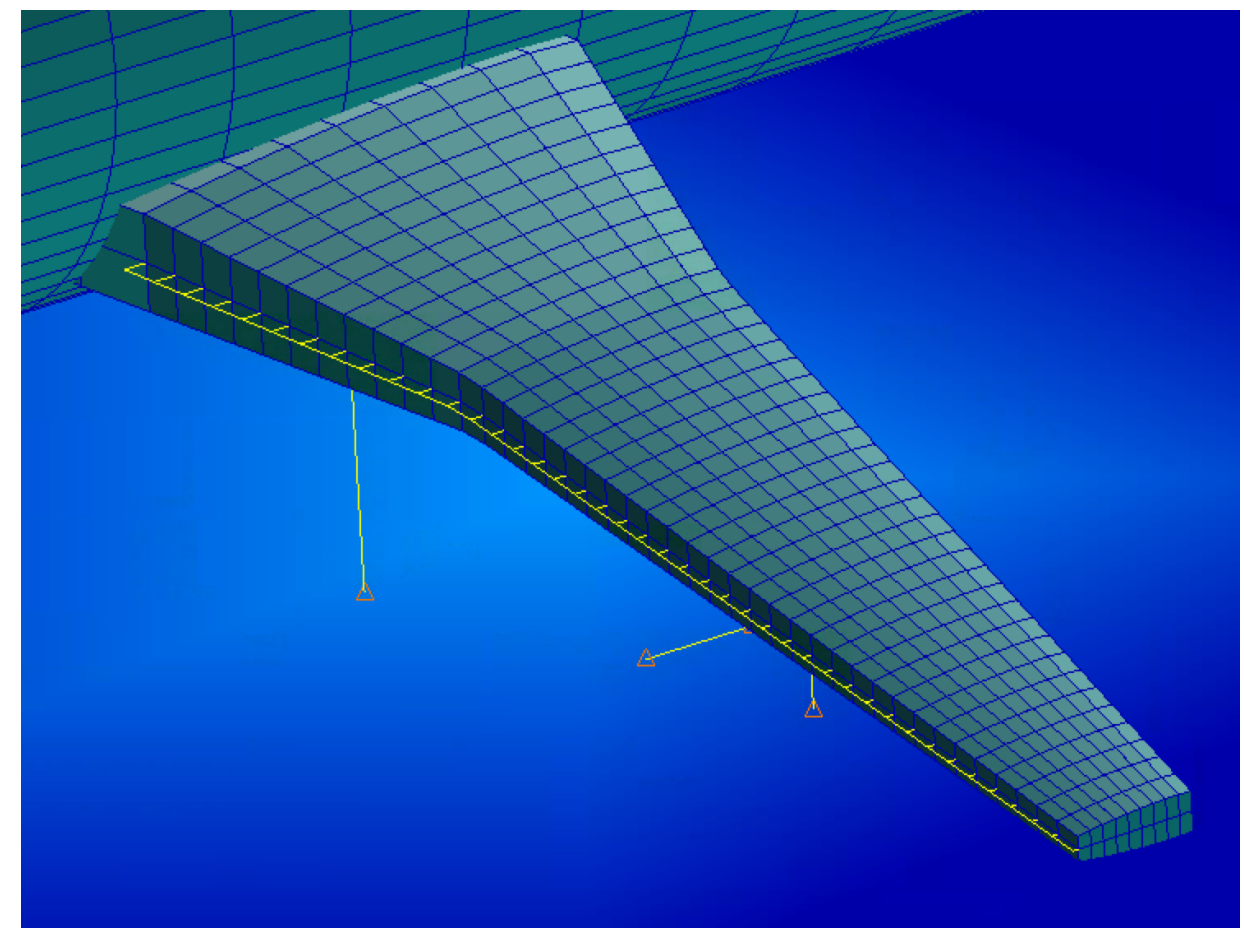

(b) Distributed ailerons example.

Fig. 2 Two example aileron structural models configured using HCDstruct for a conventional wing planform. In (a), a single outboard aileron is shown, whereas in (b) eight ailerons are shown distributed along the length of the wing. Note that the aerodynamic panels are removed for clarity. 
Table 1 Summary of Input Required for HCDstruct to Construct a Complete Set of Flutter Analysis Bulk Data Files for Execution by Nastran

\begin{tabular}{|c|c|c|c|}
\hline Parameter & Input Flag & Example Value & Comment \\
\hline Reference chord & refc & 245.0 & Reference chord length required for Nastran AERO input \\
\hline Reference density & refrho & $2.36 \mathrm{E}-5$ & Reference density required for Nastran AERO input \\
\hline Eigenvalue extraction method & eigMethod & MGIV & Required for eigenvalue analysis via Nastran EIGR input \\
\hline Number of eigenvalues & nEig & 10 & Desired number of eigenvalues for eigenvalue analysis via Nastran EIGR input \\
\hline Flutter method & flutterMethod & PK & Flutter analysis method via Nastran FLUTTER input \\
\hline Number of output eigenvalues & nEigOut & 5 & Number of eigenvalues to be printed in the Nastran F06 file \\
\hline Reduced frequencies & redFreqList & $0.001,0.05,0.1,0.2$ & List of reduced frequencies desired for flutter analysis; limited to 8 entries \\
\hline Density ratios & densityList & 1., 1.1 & List of density ratios desired for flutter analysis; limited to 8 entries \\
\hline Velocities & velocityList & 4800.0, 6000.0, & List of velocities desired for flutter analysis; unlimited number of entries \\
\hline Flutter modes & Imodes & 10 & Number of modes to be used in the flutter analysis \\
\hline Velocity scaling & vref & 12.0 & Factor to scale output velocities in the Nastran F06 file \\
\hline
\end{tabular}




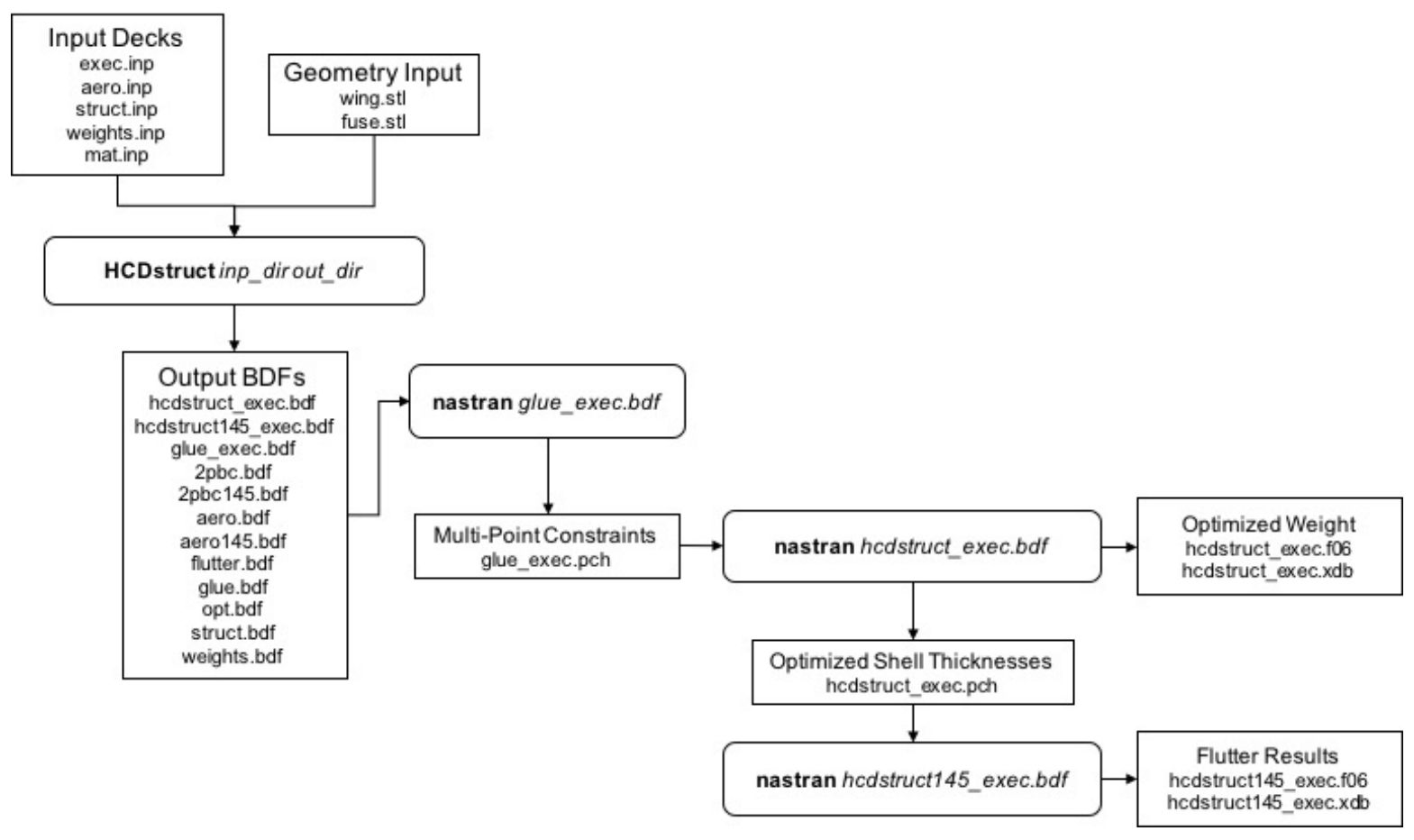

Fig. 3 Flowpath for executing flutter analysis using HCDstruct beginning with input and geometry files.

\section{Process Verification}

The baseline Boeing 737-200 model used to verify earlier static aeroelastic analysis developments has been used here to verify the SOL 145 implementation. Complete details of this model can be found in Ref. [3]. A three-view of the 737-200 Open Vehicle Sketch Pad (OpenVSP)[17] geometric model is shown in Fig. 4 for reference. The primary objective of the current 737-200 analysis was to verify the implementation of the control surface models and SOL 145 support. Example flutter analysis results for the 737-200 main wing will be presented in Section III.B.

\section{A. Aerostructural Model}

A three-dimensional aerostructural finite-element model (FEM) of the 737-200 is presented in Fig. 5, where the primary structural elements and aerodynamic model panels are rendered, along with the rigid bars and concentrated masses for modeling the empennage, propulsion system, and gear inertial loads. The model consists of 3869 CQUAD4 shell elements, 43 RBAR1 rigid bar elements, 70 CONM2 concentrated masses, 642 CAERO1 doublet-lattice method (DLM) panels, and 40 CAERO2 slender body element stations. Additional details of this model can be found in Refs. [2] and [3], including the static aeroelastic structural weight optimization used to size the shell elements, referenced below.

Since the Boeing 737-200 is designed to be unsusceptible to flutter, calculating relevant flutter characteristics is unwarranted for the purposes of demonstrating the process described in Section II.B. Rather, the goal of the flutter analysis described here is to demonstrate the application of the process implemented in HCDstruct for the same baseline configuration used to verify earlier static aeroelastic methods. To this end, the main wing was isolated from the 737-200 aerostructural model shown in Fig. 5 for the current study to verify implementation of the HCDstruct flutter analysis procedure, as some model features implemented for the static aeroelastic studies are not suitable for dynamic aeroelastic simulations, such as the rigid bar connections of the empennage, landing gear, and engines. The wing aerostructural model consists of 2017 CQUAD4 shell elements and 450 CAERO1 DLM panels, which were splined to the front and rear wing spars. The shell element thicknesses were specified using the static aeroelastic structural optimization results found in Ref. [2], and the model was constrained using SPC specifications at the centerline nodes.

\section{B. Results}

SOL 145 analysis of the Boeing 737-200 wing model described above was performed using the PK analysis method. The results of this analysis are shown in this section and have not been validated using experimental or simulation data, 


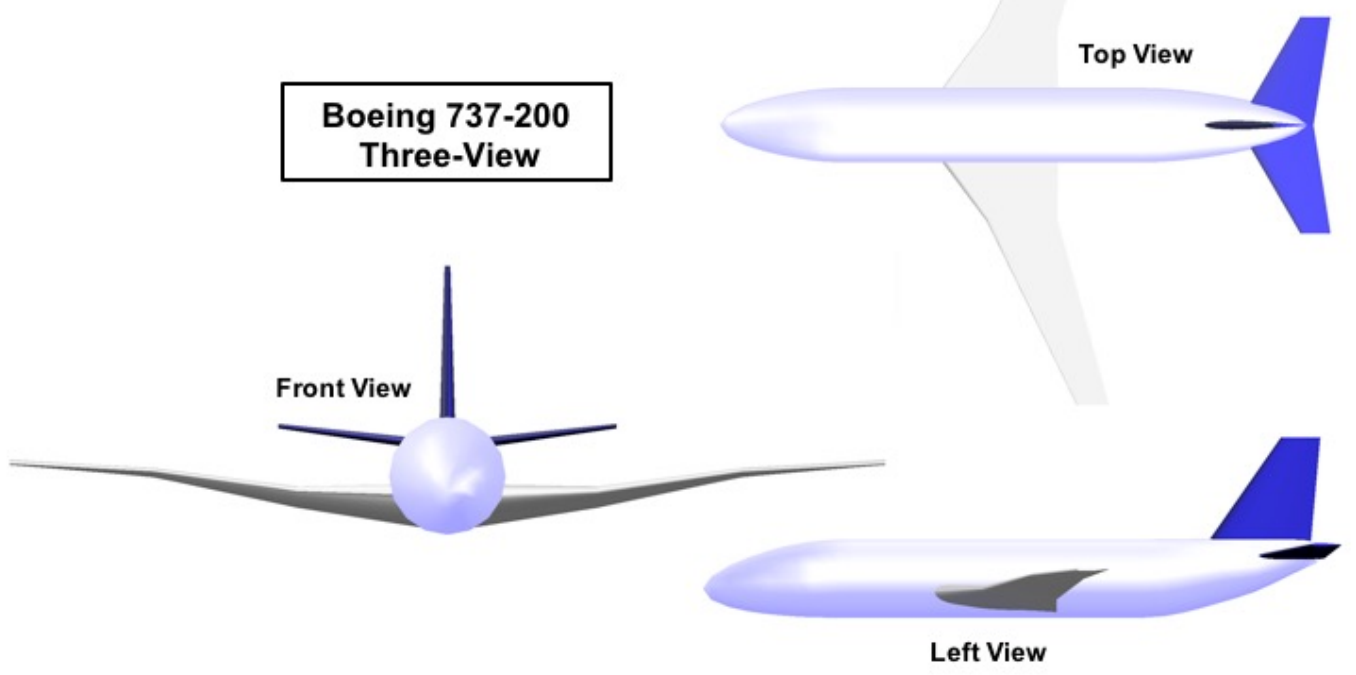

Fig. 4 A three-view of the 737-200 model rendered using OpenVSP.

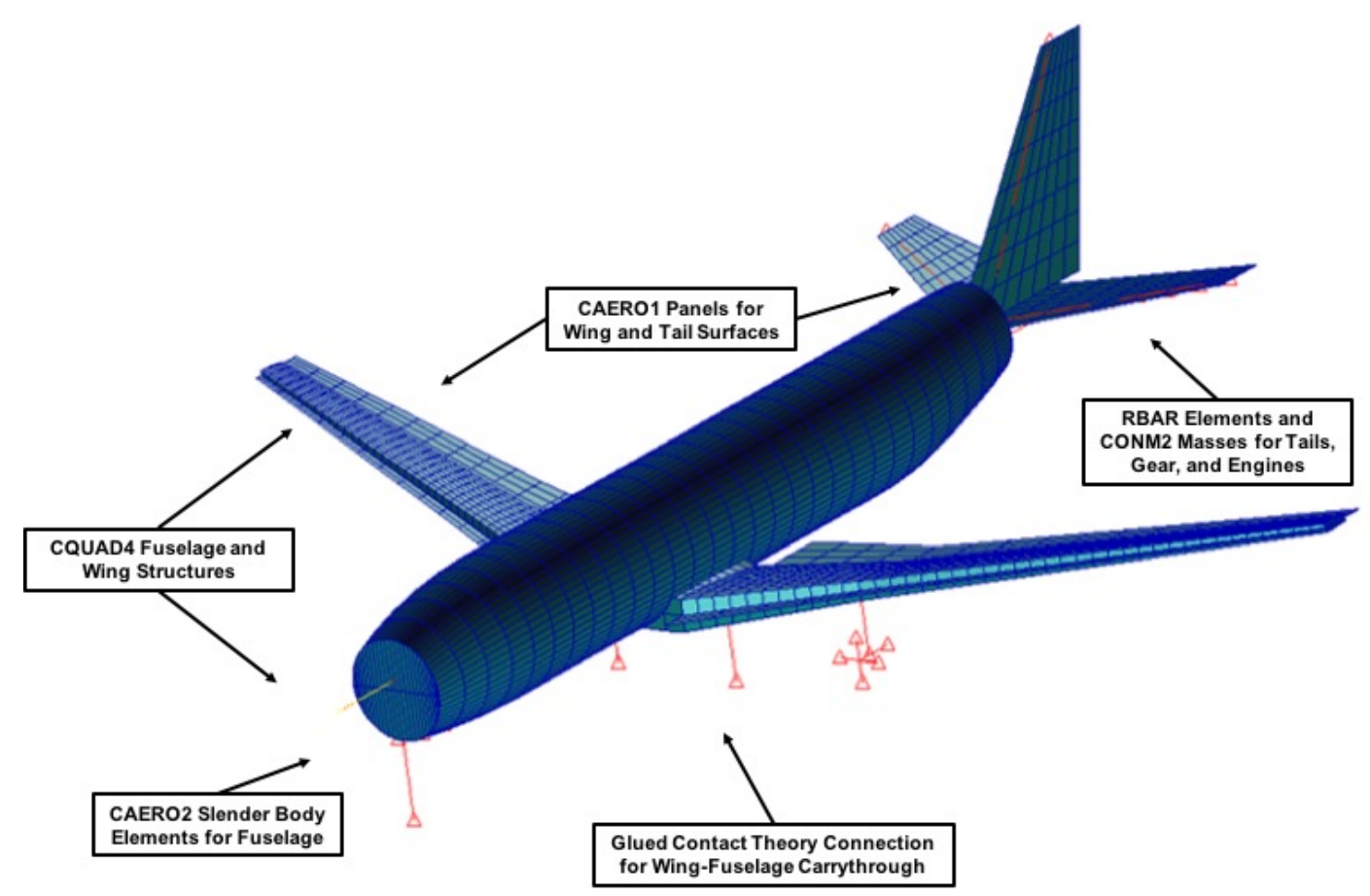

Fig. 5 An annotated depiction of the 737 aerostructural FEM showing relevant model features. Note that the slender body elements used to aerodynamically model the fuselage cannot be rendered by MSC Patran/FlightLoads.[18] 


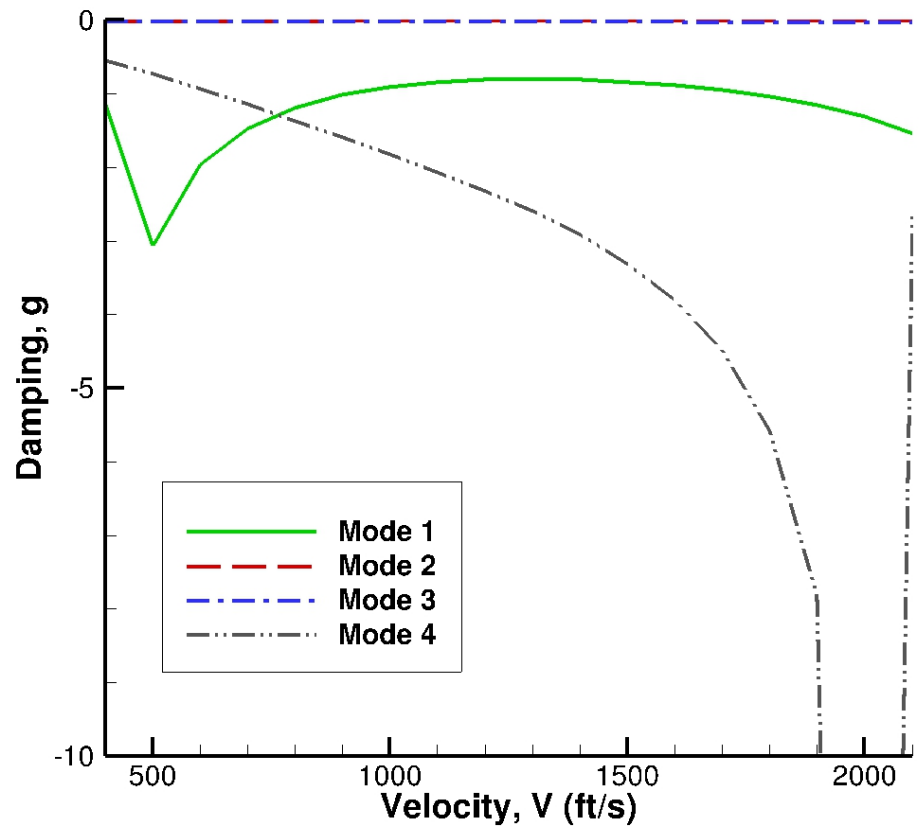

Fig. 6 Damping coefficient, $g$, as a function of velocity, $V$ ( $\mathrm{ft} / \mathrm{s})$, for the first four modes of the Boeing 737-200 wing analysis.

as none could be found in the open literature; rather, these results are shown to provide an example of the data that can be readily obtained using HCDstruct with SOL 145. For this analysis, the Mach number was held fixed at 0.0 (no Prandtl-Glauert correction), and the density was held constant at $2.36 \mathrm{E}-5 \mathrm{lbm} / \mathrm{in}^{3}$, corresponding to a standard atmosphere at $20,000 \mathrm{ft}$ altitude. The reduced frequency range was set to $(0.001,0.05,0.10,0.20,0.50,1.0)$, and the velocity (true airspeed) range included $400 \mathrm{ft} / \mathrm{s}$ to $2100 \mathrm{ft} / \mathrm{s}$ in $100 \mathrm{ft} / \mathrm{s}$ increments. The 15 lowest eigenvalue normal modes were used for the flutter analysis, and a structural mode damping factor of 0.01 was assumed.

Damping coefficient $(g)$ and flutter frequency $(f)$ results from the Nastran F06 flutter summary are plotted as a function of velocity $(V)$ in Figs. 6 and 7, respectively. The $V$-g curves remain negative across all velocities, indicating no onset of flutter at the investigated conditions, and the $V-f$ curves show that the first four flutter modes vary in frequency from approximately 1 to $29 \mathrm{~Hz}$. Additionally, for reference, the accompanying first four flutter mode shapes are shown in Fig. 8 for a velocity of $1000 \mathrm{ft} / \mathrm{s}$, where the first, second, and third modes correspond to the first, second, and third bending modes of the wing. The fourth mode appears to represent the second chordwise-bending mode for the wing; the first chordwise-bending mode is assumed to be outside the velocity range investigated here.

\section{Summary and Future Work}

This paper provides an update on the continuing development of a flutter analysis capability for HCDstruct. The first step toward this new capability was extending the control surface models within HCDstruct to include structural representations capable of modeling the actuator and control surface dynamics. With this completed, direct support for Nastran's SOL 145 flutter analysis solution sequence was implemented. This development required significant modifications of the input files and the source code to process the configuration data supplied by the user in order to build a complete set of SOL 145 BDFs for Nastran execution. The first demonstration of this new capability was presented in Section III, where the baseline Boeing 737-200 model used to verify the static aeroelastic weight optimization methods implemented in HCDstruct was used to perform flutter analysis on the main wing using the PK method. This analysis yielded $V-g$ and $V-f$ results for the requested flutter modes, which can be readily used to surmise conditions of flutter onset. The results shown in Section III.B are meant only to demonstrate the applicability of this new capability; validation of this new capability is an ongoing effort, described in more detail below. 


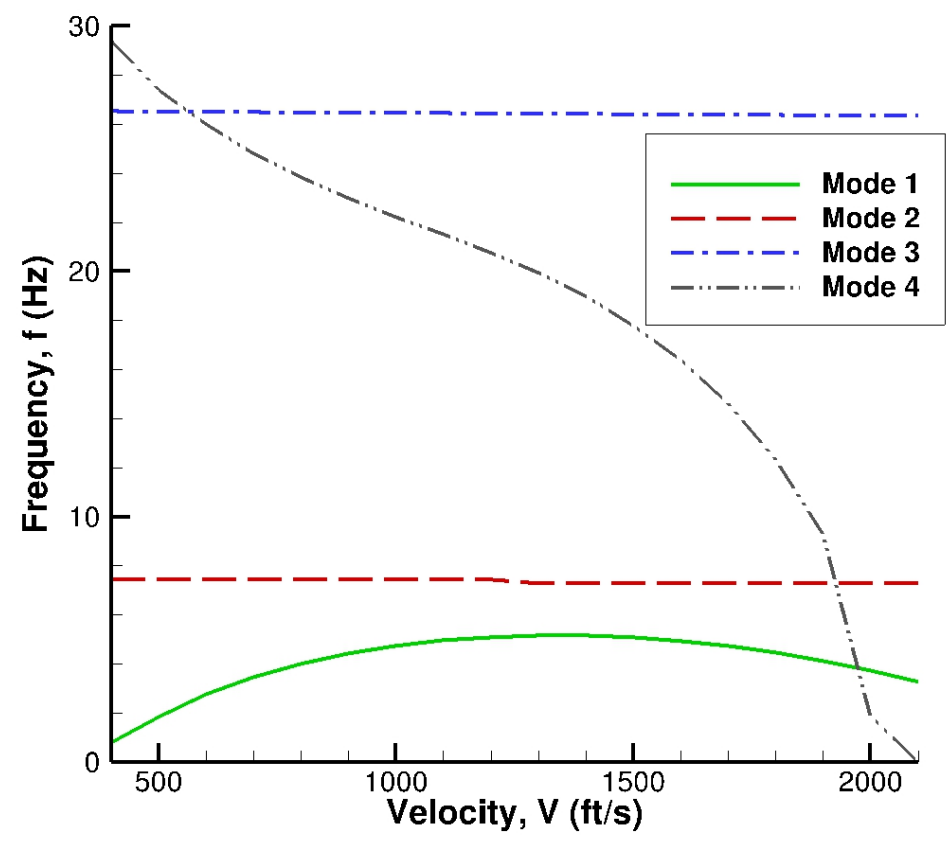

Fig. 7 Frequency, $f(\mathrm{~Hz})$, as a function of velocity, $V(\mathrm{ft} / \mathrm{s})$, for the first four modes of the Boeing 737-200 wing analysis.

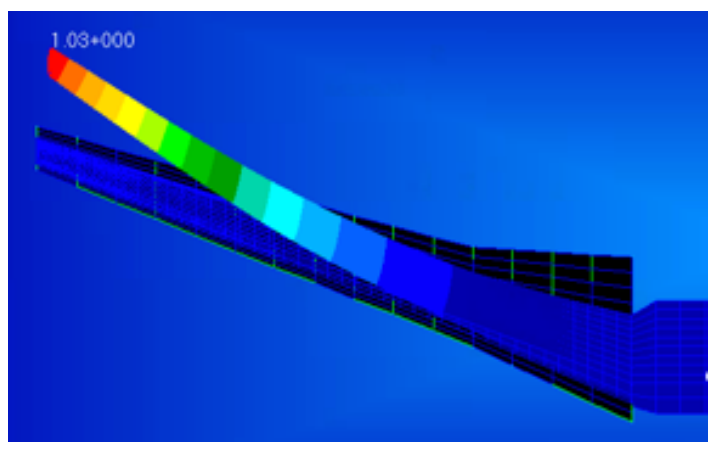

(a) Flutter mode one.

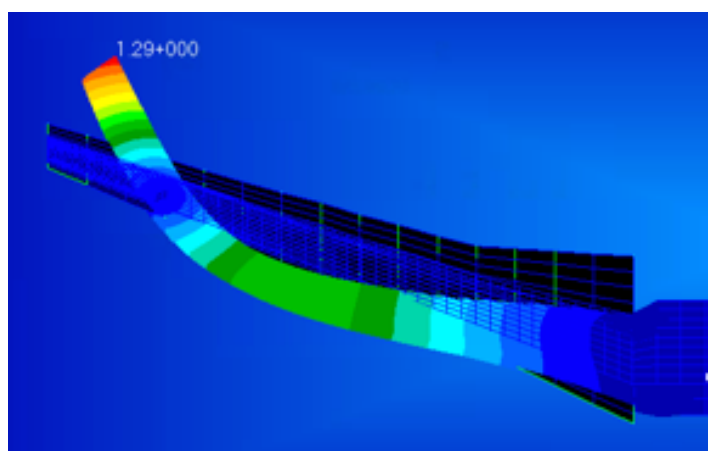

(d) Flutter mode four.

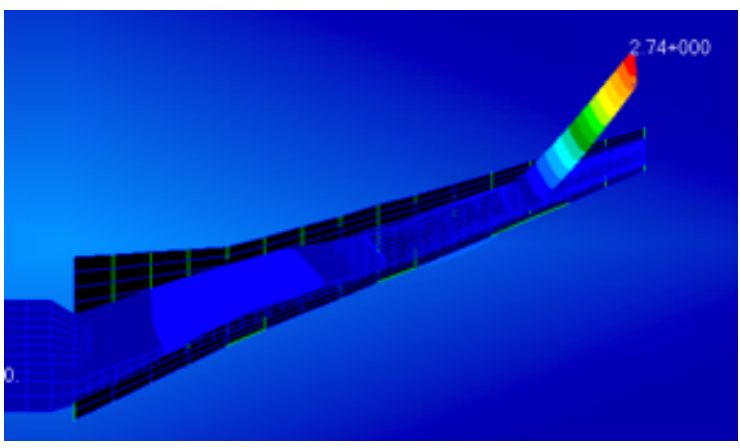

(b) Flutter mode two.

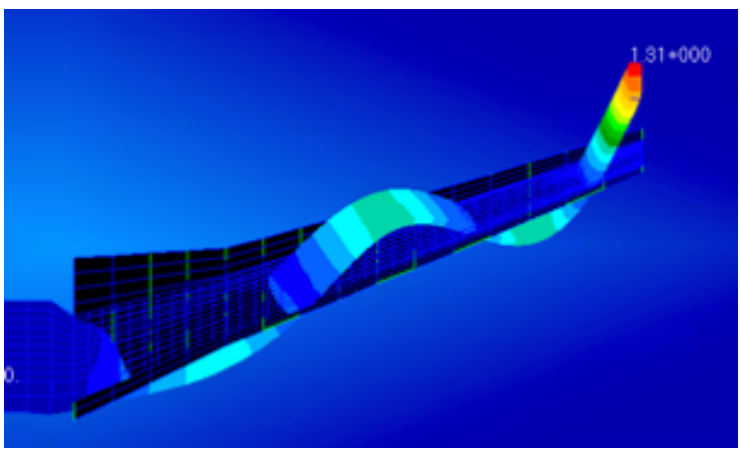

(c) Flutter mode three.

Fig. 8 First four flutter modes for the Boeing 737-200 wing model. Note that only half of each model and mode shape is shown, as both are symmetric over the spanwise centerplane. 
Before application to a complete aircraft configuration is pursued, validation of the flutter analysis methodology implemented in HCDstruct will be completed using available test cases from the open literature. Specifically, future work includes performing a validation study using the High Reynolds Number Aero-Structural Dynamics (HiRENASD) model test case[19] from the AIAA Aeroelastic Prediction Workshop (AePW)[20]. The HiRENASD model is an experiment performed in the European Transonic Windtunnel (ETW), which utilized a $34^{\circ}$ aft-swept, tapered clean wing with a BAC 3-11 supercritical airfoil profile[21]. Extensive experimental data is available at Mach 0.7 and 0.8, which provides an opportunity to validate the SOL 145 analysis support implemented in HCDstruct in conjunction with the aerodynamic-matching utility, aeroMatch, published in Ref. [2]. Additional validation cases will include the Rectangular Supercritical Wing (RSW) and Baseline SuperCritical Wing (BSCW) AePW test cases[21]. Once validation is complete, HCDstruct will be applied to complete aeroelastic models of advanced aircraft concepts of particular interest, including NASA STARC-ABL, NASA D8, and Transonic Truss-Braced Wing (TTBW) configurations studied in recent years by NASA.

\section{Acknowledgments}

This work was supported by NASA's Transformational Tools and Technologies (TTT) Project, within the MultiDisciplinary Analysis and Optimization element, led by Mrs. Patricia Glaab. The authors thank Dr. Erik Olson for his assistance in reviewing this manuscript.

\section{References}

[1] McCullers, L. A., FLOPS Flight Optimization System, FLOPS Users Manual, December 2009.

[2] Quinlan, J., and Gern, F. H., "Extension of HCDstruct for Transonic Aeroservoelastic Analysis of Unconventional Aircraft Concepts," AIAA AVIATION Forum, AIAA 2017-4379, Denver, CO, 2017.

[3] Quinlan, J. R., and Gern, F. H., "Aeroelastic Optimization of Generalized Tube and Wing Aircraft Concepts using HCDstruct Version 2.0,” AIAA SciTech Forum and Exposition, AIAA 2017-0202, Dallas, TX, 2017.

[4] Quinlan, J. R., and Gern, F. H., "Optimization of an Advanced Hybrid Wing Body Concept using HCDstruct Version 1.2," AIAA Aviation Conference, AIAA 2016-3460, Washington, DC, 2016.

[5] Gern, F. H., "Update on HCDstruct-A Tool for Hybrid Wing Body Conceptual Design and Structural Optimization," 15th AIAA Aviation Technology, Integration, and Operations Conference, AIAA 2015-2544, Dallas, TX, 2015.

[6] Li, W., and Robinson, J., "Automated Generation of Finite-Element Meshes for Aircraft Conceptual Design," AIAA AVIATION Forum, AIAA 2016-3287, Washington, DC, 2016.

[7] Chambers, J. T., Yutko, B. M., Singh, R., and Church, C., "Structural Optimization Study of the D8 Double-Bubble Composite Fuselage," AIAA SciTech Forum, AIAA 2017-0508, Grapevine, TX, 2017.

[8] Kiviaho, J. F., Jacobson, K., Smith, M. J., and Kennedy, G., "A Robust and Flexible Coupling Framework for Aeroelastic Analysis and Optimization,” AIAA AVIATION Forum, AIAA 2017-4144, Denver, CO, 2017.

[9] Corman, J. A., Rancourt, D., Lee, C., Mavris, D. N., and Wilson, T., "Preliminary Wing Weight Estimation Under Probabilistic Loads for a Transport Aircraft," AIAA SciTech Forum, AIAA 2014-1205, National Harbor, MD, 2014.

[10] Corman, J. A., and Mavris, D. N., "Characterization of an Aero-Structural Interaction for the Hybrid Wing Body Center Section in Conceptual Phase Structural Sizing,” AIAA AVIATION Forum, AIAA 2014-3159, Atlanta, GA, 2014.

[11] Nigam, N., Ayyalasomayajula, S. K., Qi, X., Chen, P. C., and Alonso, J. J., "High-Fidelity Weight Estimation for Aircraft Conceptual Design Optimization," AIAA AVIATION Forum, AIAA 2015-3360, Dallas, TX, 2015.

[12] MSC Nastran 2008, Quick Reference Guide, MSC Software Corporation, Santa Ana, CA, 2008.

[13] Bradley, K. R., “A Sizing Methodology for the Conceptual Design of Blended-Wing-Body Transports,” Tech. rep., NASA/CR2004-213016, NASA Langley Research Center, Hampton, VA, September 2004.

[14] Li, V., and Velicki, A., "Advanced PRSEUS Structural Concept Design and Optimization," 12th AIAA/ISSMO Multidisciplinary Analysis and Optimization Conference, AIAA 2008-5840, Victoria, British Columbia, Canada, 2008. 
[15] Jadic, I., Hermann, T., Hartley, D., and Giri, J., "Generalized Aerodynamic Forces based on CFD and Correction Factor Techniques for AGARD Wing 445.6," AIAA/ASME/ASCE/AHS/ASC Structures, Structural Dynamics and Materials Exhibit, AIAA 2001-1208, Seattle, WA, 2001.

[16] Moreno, R., Fedime, K., Narisetti, R., and Taylor, P. F., "A Modification to the Enhanced Correction Factor Technique to Correlate with Experimental Data," AIAA/ASME/ASCE/AHS/ASC Structures, Structural Dynamics and Materials Exhibit, AIAA 2015-1421, Kissimmee, FL, 2015.

[17] Hahn, A., "Vehicle Sketch Pad: A Parametric Geometry Modeler for Conceptual Aircraft Design," 48th AIAA Aerospace Sciences Meeting and Exhibit, AIAA 2010-657, Orlando, FL, 2010.

[18] MSC FlightLoads 2006, User's Guide, MSC Software Corporation, Santa Ana, CA, 2006.

[19] Ballman, J., Dafnis, A., Korsch, H., Buxel, C., Reimerdes, H.-G., Brakhage, K.-H., Braun, C., Baars, A., and Boucke, A., "Experimental Analysis of High Reynolds Number Aero-Structural Dynamics in ETW," AIAA Aerospace Sciences Meeting and Exhibit, AIAA 2008-841, Reno, NV, 2008.

[20] Heeg, J., Chwalowski, P., Schuster, D., and Dalenbring, M., "Overview and Lessons Learned from the Aeroelastic Prediction Workshop," AIAA/ASME/ASCE/AHS/ASC Structures, Structural Dynamics, and Materials Conference, AIAA 2013-1798, Boston, MASS, 2013.

[21] Heeg, J., Chwalowski, P., and Florance, J. P., "Overview of the Aeroelastic Prediction Workshop,” AIAA Aerospace Sciences Meeting, AIAA 2013-783, Grapevine, TX, 2013. 